\title{
LETTER TO THE EDITOR Practice-relevant revision of IPSET-thrombosis based on 1019 patients with WHO-defined essential thrombocythemia
}

Blood Cancer Journal (2015) 5, e369; doi:10.1038/bcj.2015.94; published online 27 November 2015

Current risk stratification for thrombosis in essential thrombocythemia (ET) is two-tiered and considers low- and high-risk categories based on the respective absence or presence of either age $>60$ years or history of thrombosis. ${ }^{1}$ In the recent International Prognostic Score for Thrombosis in ET (IPSETthrombosis), age and history of thrombosis were confirmed as independent risk factors for future thrombosis and the study also identified independent prothrombotic role for cardiovascular (CV) risk factors and JAK2V617F mutation. ${ }^{2}$ This model outperformed the two-tiered conventional risk stratification in predicting future vascular events and was not further affected by the recently discovered CALR mutation. ${ }^{3}$ In the current study, we re-analyzed the original IPSET-thrombosis data in 1019 patients with WHOdefined ET in whom JAK2 mutational status was available, to quantify the individual contributions of JAK2 mutations and CV risk factors in conventionally assigned low- and high-risk ET.

After approval from their respective institutional review boards, seven centers from Italy, Austria and the United States, belonging to the International Working Group for myeloproliferative neoplasm (MPN) Research and Treatment (IWG-MRT), collectively submitted diagnostic and follow-up information on 1220 patients, locally diagnosed with 'WHO-defined $\mathrm{ET}^{\prime}{ }^{4}$ Among these, 1019 patients were selected in whom JAK2 mutational status was available. Objectively proven major arterial and venous events ${ }^{2}$ were reported as rates per 100 patient-years and the difference among groups was assessed by Mantel Cox log-rank test. The Kaplan-Meier product-limit method was used to estimate thrombosis-free survival curves, and the log-rank test was adopted to compare survival curves.

At diagnosis, conventionally assigned low-risk and high-risk groups were significantly different in terms of the frequency of CV risk factors $(P<0.001)$ and JAK2 mutational status $(P<0.001)$.

Median follow-up was 6.8 and 5.0 years in conventionally assigned low- and high-risk patients, respectively. Low-dose aspirin and cytoreduction drugs (Hydroxyurea in around 85\%) were prescribed in 58 and $41 \%$ in low-risk and in 71 and $81 \%$ in high risk, respectively. In low-risk patients cytoreduction was started after a median of 18 months since diagnosis because of occurrence of vascular events, age rising to 60 years or progressive thrombocytosis. The overall annual rate of total thrombosis (108 events) in conventionally assigned low- and high-risk patients was $1.11 \%$-pt/y (confidence interval $(\mathrm{Cl}) 0.81-1.52$ ) and $2.46 \%$-pt/y (Cl 1.94-3.11), respectively $(P=0.001)$, and the difference was mainly due to a higher frequency of arterial thrombosis in highrisk patients $(P<0.001)$.

The influence of JAK2 mutations and CV risk factors on the rate of thrombosis in conventionally assigned low- and high-risk groups is presented in the table.

(i) Conventionally assigned low-risk group. Amongst 506 patients, $200(40 \%)$ displayed neither JAK2 mutation nor CV risk factors and their annual rate of thrombosis was $0.44 \%$, as opposed to $1.05 \%$ in the presence of CV risk factors ( $P=$ not significant (NS)), $1.59 \%$ in the presence of JAK2 mutation $(P=0.001)$ and $2.57 \%$ in the presence of both CV risk factors and JAK2 mutation $(P<0.001)$. There was no significant difference when low-risk patients with both JAK2 mutation and CV risk factors were compared with those with JAK2 mutation only $(P=0.217)$. Figure 1 a shows the time to major thrombosis among patients with the absence or presence of one or two additional risk factors (that is, JAK2 mutations and CV risk factors).

(ii) Conventionally assigned high-risk group: the absence or presence of one or both of the aforementioned additional risk factors for thrombosis were documented in 111 (22\%), 44 (9\%), $222(43 \%)$ and $136(27 \%)$ patients, respectively, with corresponding annual rates of thrombosis at 1.44, 1.64, 2.36 and $4.17 \%$ (Table). High-risk patients with both risk factors had a significantly higher risk of thrombosis compared with their counterparts without JAK2 mutations and CV risk factors $(P=0.011)$. Figure 1 b shows the probability of events in the groups.

Additional analysis revealed limited enhancement of thrombosis risk by either JAK2 mutations or CV risk factors or both in patients whose high-risk status was defined by the presence of thrombosis history, regardless of age $(P=N S)$. In contrast, the presence of JAK2 mutations, with or without CV risk factors, might have affected thrombosis risk in patients where high-risk status was defined by age alone $(P=0.05)$.

The current study quantifies the individual and combined risk contribution of CV risk factors and JAK2 mutation in both conventionally defined low- and high-risk ET. The impressively low risk of thrombosis in low-risk JAK2-unmutated patients with $(1.05 \%$ patients/year) or without $(0.44 \%$ patients/year) CV risk factors clearly distinguishes them from conventionally assigned 'low-risk' patients with JAK2 mutations, with or without CV risk factors. It is therefore reasonable to further risk-stratify conventionally assigned 'low-risk' ET into 'very low risk' and 'low risk' categories, based on the respective absence or presence of JAK2 mutations (Figure 1c), with the caveat that 'very low risk' disease without CV risk factors is therapeutically approached differently than 'very low risk' disease with CV risk factors. Such distinction is practically relevant because aspirin therapy might not be necessary in the former group, whereas its efficacy in the latter group and in those with 'low risk' disease as suggested in a recent retrospective study ${ }^{5}$ might have been undermined by the lack of $24 \mathrm{~h}$ therapeutic coverage from the standard once-daily aspirin, especially in JAK2-mutated patients with or without CV risk factors. ${ }^{6,7}$

In high-risk ET, among the two conventional risk factors, the current study delineates thrombosis history as being significantly more detrimental than advanced age. Furthermore, the effect of JAK2 mutations and CV risk factors in high-risk disease was more apparent, although not statistically significant, in patients whose high-risk disease status was determined by advanced age, whereas the additional impact of these additional risk factors was not significant in high-risk patients with history of thrombosis. This suggests the possible consideration of older patients without thrombosis history or JAK2 mutations as 'intermediate-risk' and reserve the 'high-risk' label to patients with thrombosis or to those who are $>60$ years but also display JAK2 mutations. In other words, the revised risk stratification scheme might include 
a

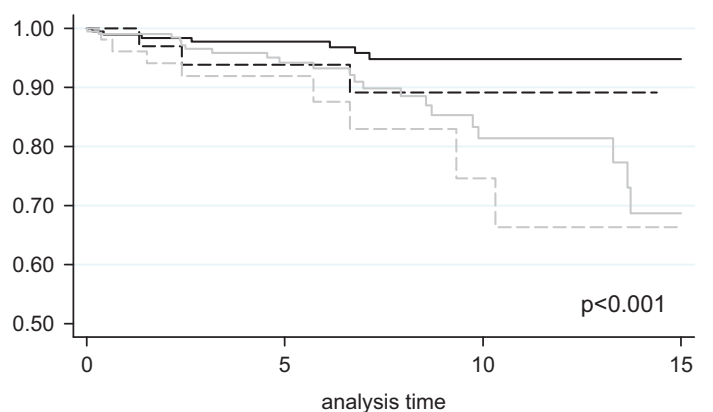

b

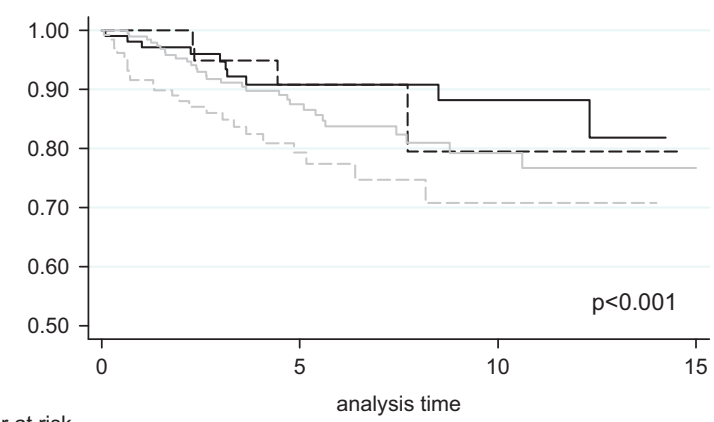

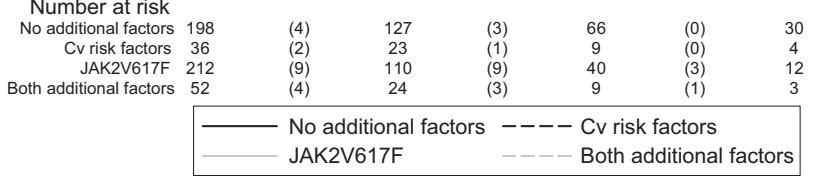

Number at risk

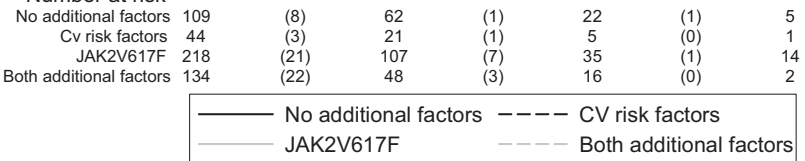

C

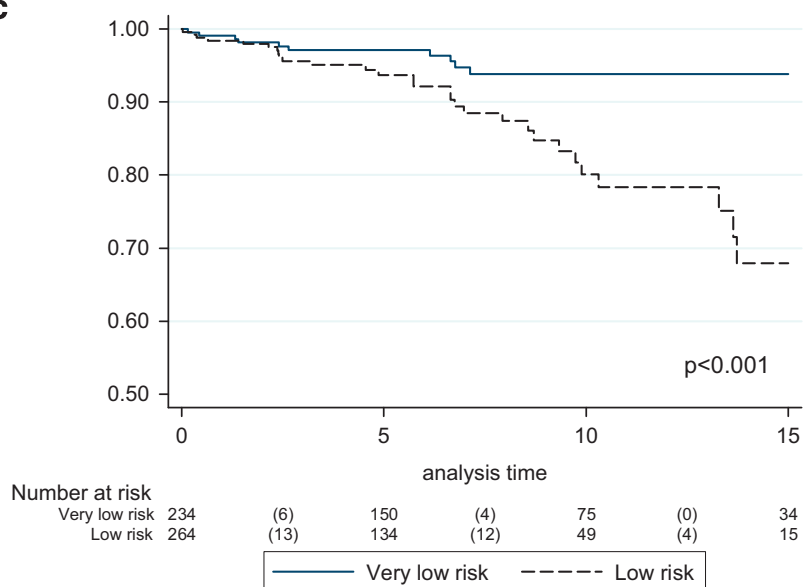

d

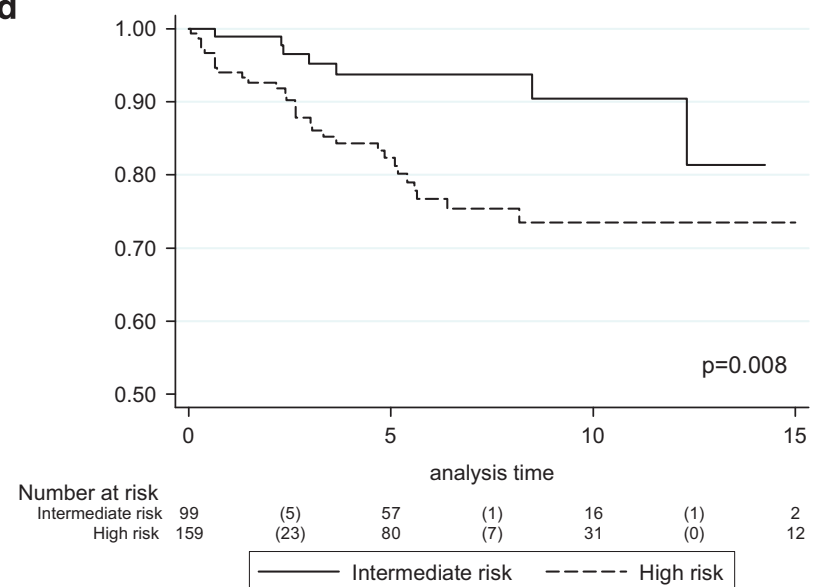

Figure 1. Thrombosis-free survival in conventionally defined low- and high-risk (a and $\mathbf{b}$ ) and in the revised risk classification (c and d). (a) Low-risk patients with or without additional risk factors (CV risk factors and JAK2 mutation); (b) High-risk patients with or without additional risk factors (CV risk factors and JAK2 mutation); (c) 'Very low risk' (no thrombosis history, age $\leqslant 60$ years and JAK2-unmutated): 'Low risk' (no thrombosis history, age $\leqslant 60$ years and JAK2-mutated); (d) 'Intermediate risk' (no thrombosis history, age >60 years and JAK2unmutated); 'High risk' (thrombosis history or age $>60$ years with JAK2 mutation).

Table 1. Influence of cardiovascular risk factors and JAK2 mutation on the rate of vascular events in low- and high-risk patients

\begin{tabular}{|c|c|c|c|c|c|c|}
\hline Low risk & $506(50)$ & & & & & \\
\hline None & $200(40)$ & 7 & $0.44(0.21-0.92)$ & Ref & & \\
\hline JAK2V617F & $213(43)$ & 21 & 1.59 (1.04-2.44) & 0.001 & 0.217 & \\
\hline Both & $52(10)$ & 8 & $2.57(1.29-5.15)$ & $<0.001$ & Ref & $<0.001$ \\
\hline High risk & $513(50)$ & & & & & \\
\hline JAK2V617F & $222(43)$ & 30 & $2.36(1.65-3.38)$ & 0.168 & 0.082 & \\
\hline Both & $136(27)$ & 25 & $4.17(2.82-6.17)$ & 0.011 & Ref & 0.005 \\
\hline
\end{tabular}

four categories: 'very low risk' (no thrombosis history, age $\leqslant 60$ years and JAK2-unmutated); 'low risk' (no thrombosis history, age $\leqslant 60$ years and JAK2-mutated); intermediate risk' (no thrombosis history, age $>60$ years and JAK2-unmutated) and high risk (thrombosis history or age $>60$ years with JAK2 mutation). Figures $1 \mathrm{c}$ and $\mathrm{d}$ show the thrombosis-free survival probability of patients according to this revised risk stratification.

Treatment recommendations for each one of the abovementioned new risk categories should be examined in the context of prospective controlled studies. Until results from controlled studies become available, we would not insist on the use of aspirin in 'very low risk' disease without CV risk factors while we advise once-daily aspirin in 'very low risk' disease with CV risk factors. We believe that it is reasonable, but not mandated, to consider twice-daily aspirin in 'low-risk' JAK2-mutated patients, especially in the presence of CV risk factors. Similarly, although we encourage the use of cytoreductive therapy in both 'intermediate risk' and 'high risk' disease, we would not insist in its use in 'intermediaterisk' patients, who could be treated, instead, with twice-daily aspirin. 


\section{CONFLICT OF INTEREST}

The authors declare no conflict of interest.

\section{ACKNOWLEDGEMENTS}

TB, AMV and AR were supported by a grant from the Associazione Italiana per la Ricerca sul Cancro (Milan, Italy), Special Program Molecular Clinical Oncology $5 \times 1000$ to the Italian Association for Cancer Research (Associazione Italiana per la Ricerca sul Cancro) and Gruppo Italiano Malattie Mieloproliferative (project number 1005). A detailed description of the project is available at http://www.progettoagimm.it.

T Barbui $^{1,2}$, AM Vannucchi $^{3}, \mathrm{~V}$ Buxhofer-Ausch ${ }^{4}, \mathrm{~V}$ De Stefano ${ }^{5}$, S Betti ${ }^{5}$, A Rambaldi $^{2}$, E Rumi $^{6}$, M Ruggeri $^{7}$, F Rodeghiero $^{7}$, ML Randi ${ }^{8}$, I Bertozzi ${ }^{8}$, H Gisslinger ${ }^{4}$, G Finazzi ${ }^{2}$, A Carobbio ${ }^{1}$ J Thiele ${ }^{9}$, F Passamonti $^{10}$, C Falcone $^{1}$ and A Tefferi ${ }^{11}$

${ }^{1}$ Research Foundation, Papa Giovanni XXIII Hospital, Bergamo, Italy; ${ }^{2}$ Division of Hematology, Papa Giovanni XXIII Hospital, Bergamo, Italy;

${ }^{3}$ Department of Experimental and Clinical Medicine, University of Florence, Florence, Italy; ${ }^{4}$ Department of Internal Medicine I, Division of Hematology and Blood Coagulation, Medical University of Vienna, Vienna, Austria;

${ }^{5}$ Institute of Hematology, Catholic University, Rome, Italy;

${ }^{6}$ Department of Hematology Oncology,

Fondazione Istituto di Ricovero e Cura a Carattere Scientifico (IRCCS) Policlinico San Matteo, Pavia, Italy;

${ }^{7}$ Division of Hematology, S. Bortolo Hospital, Vicenza, Italy; ${ }^{8}$ Department of Medicine-DIMED, University of Padua, Padua, Italy; ${ }^{9}$ Institute for Pathology, University of Cologne, Cologne, Germany; ${ }^{10}$ University Hospital Ospedale di Circolo and Fondazione Macchi, Division of Hematology, Varese, Italy and

${ }^{11}$ Division of Hematology, Mayo Clinic, Rochester, MN, USA E-mail: tbarbui@hpg23.it

\section{REFERENCES}

1 Barbui T, Barosi G, Birgegard G, Cervantes F, Finazzi G, Griesshammer M et al. Philadelphia negative classical myeloproliferative neoplasms: critical concepts and management recommendations from European LeukemiaNet. J Clin Oncol 2011; 29: $761-770$.

2 Barbui T, Finazzi G, Carobbio A, Thiele J, Passamonti F, Rumi E et al. Development and validation of an International Prognostic Score of thrombosis in World Health Organization-essential thrombocythemia (IPSET-thrombosis). Blood 2012; 120: 5128-5133

3 Finazzi G, Carobbio A, Guglielmelli P. Calreticulin mutation does not modify the IPSET score for predicting the risk of thrombosis among 1150 patients with essential thrombocythemia. Blood 2014; 124: 2611-2612.

4 Vardiman JW, Thiele J, Arber DA, Brunning RD, Borowitz MJ, Porwit A et al. The 2008 revision of the World Health Organization (WHO) classification of myeloid neoplasms and acute leukemia: rationale and important changes. Blood. 2009 114: 937-951.

5 Alvarez-Larrán A, Cervantes F, Pereira A, Arellano-Rodrigo E, Pérez-Andreu V, Hernández-Boluda $J C$ et al. Observation versus antiplatelet therapy as primary prophylaxis for thrombosis in low-risk essential thrombocythemia. Blood 2010; 116: $1205-1210$.

6 Dillinger JG, Sideris G, Henry P, Bal dit Sollier C, Ronez E, Drouet L. Twice daily aspirin to improve biological aspirin efficacy in patients with essential thrombocythemia. Thromb Res. 2012; 129: 91-94.

7 Pascale S, Petrucci G, Dragani A, Habib A, Zaccardi F, Pagliaccia F et al. Aspirin-insensitive thromboxane biosynthesis in essential thrombocythemia is explained by accelerated renewal of the drug target. Blood 2012; 119: 3595-3603.

\section{(c) (i)}

This work is licensed under a Creative Commons Attribution 4.0 International License. The images or other third party material in this article are included in the article's Creative Commons license, unless indicated otherwise in the credit line; if the material is not included under the Creative Commons license, users will need to obtain permission from the license holder to reproduce the material. To view a copy of this license, visit http://creativecommons.org/licenses/ by/4.0/ 